\title{
Quantitative assessment of cardiovascular autonomic impairment in cancer survivors: a single center case series
}

\author{
Benjamin Noor', Shannel Akhavan², Michael Leuchter ${ }^{3}$, Eric H. Yang ${ }^{2}$ and Olujimi A. Ajijola $2,3^{*}$
}

\begin{abstract}
Background: Cardiovascular autonomic dysfunction in cancer survivors is poorly understood.

Objectives: To better characterize the clinical characteristics and types of autonomic dysfunction in this population. Methods: A retrospective analysis of cancer survivors within an academic cardio-oncology program referred for suspected autonomic dysfunction was performed. Autonomic reflex testing of adrenergic, cardiovagal, and sudomotor function was done. Autonomic impairment was graded on severity based on the Composite Autonomic Severity Score system. Patients with pre-existing autonomic dysfunction prior to their cancer diagnosis were excluded.

Results: Of approximately 282 total patients in the UCLA Cardio-Oncology program, 24 were referred for suspected autonomic dysfunction and met the inclusion criteria. 22 had autonomic impairment on autonomic reflex testing. Eight patients were female, and the mean age at time of autonomic testing was 51.3 years. The average duration from cancer diagnosis to autonomic testing was 10.3 years. The reasons for referral included dizziness, tachycardia, palpitations, and syncope. The majority of patients (75\%) had hematologic disorders. The most common chemotherapies administered were vinca alkaloids (54.2\%), alkylating agents (66.7\%), and anthracyclines (54.2\%). Most patients received radiation to the thorax (66.7\%) and neck (53.3\%). Eleven patients had mild autonomic impairment, 7 had moderate, and 4 had severe autonomic impairment. Dysfunction was commonly present in the sympathetic and parasympathetic branches, but most pronounced in the sympathetic system. The majority of patients were diagnosed with orthostatic hypotension (50\%), inappropriate sinus tachycardia (20.8\%), and postural orthostatic tachycardia syndrome (12.5\%) and had subjective improvement with treatment.

Conclusion: Cardiovascular autonomic dysfunction occurs in cancer survivors, and commonly affects both the sympathetic and parasympathetic systems. Symptom recognition in patients should prompt autonomic testing and treatment where appropriate.
\end{abstract}

Keywords: Cardiovascular autonomic dysfunction, Autonomic reflex testing, Syncope, Palpitations, Postural orthostatic tachycardia, Orthostatic hypotension, Inappropriate sinus tachycardiac, Anthracyclines, Vinca alkaloids, Alkylating agents

\footnotetext{
* Correspondence: oajijola@mednet.ucla.edu

${ }^{2}$ UCLA Cardio-Oncology Program and Division of Cardiology, Department of

Medicine, University of California at Los Angeles, Los Angeles, CA, USA

${ }^{3}$ UCLA Cardiac Arrhythmia and Neurocardiology Research Center, David

Geffen School of Medicine at UCLA, University of California at Los Angeles,

100 Medical Plaza, Suite 660, Westwood Blvd, Los Angeles, CA 90095-1679,

USA

Full list of author information is available at the end of the article
}

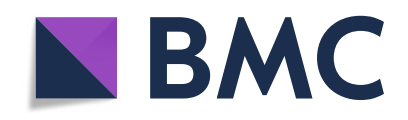

(- The Author(s). 2020 Open Access This article is licensed under a Creative Commons Attribution 4.0 International License, which permits use, sharing, adaptation, distribution and reproduction in any medium or format, as long as you give appropriate credit to the original author(s) and the source, provide a link to the Creative Commons licence, and indicate if changes were made. The images or other third party material in this article are included in the article's Creative Commons licence, unless indicated otherwise in a credit line to the material. If material is not included in the article's Creative Commons licence and your intended use is not permitted by statutory regulation or exceeds the permitted use, you will need to obtain permission directly from the copyright holder. To view a copy of this licence, visit http://creativecommons.org/licenses/by/4.0/. The Creative Commons Public Domain Dedication waiver (http://creativecommons.org/publicdomain/zero/1.0/) applies to the data made available in this article, unless otherwise stated in a credit line to the data. 


\section{Background}

Long term cancer survival rates have increased largely due to the improvements in detection and treatment of malignancies, increased detection of less malignant cancers, and advancements in the management of non-cancer-related health conditions. The five-year relative survival rate for all cancers has increased from $49 \%$ in $1975-1977$ to $69 \%$ in 2008-2014 [1]. By 2030, the population of cancer survivors in the United States is estimated to be at least 22.1 million [2]. Because of prolonged survival, long-term sequalae of cancer and cancer-directed treatments have increased in prevalence [3]. One known, but poorly understood, comorbidity in cancer survivors is cardiovascular autonomic dysfunction [4-6].

Cardiovascular autonomic impairment has been detected in $52-81 \%$ of patients with advanced cancer [7-10]. Due to an imbalance in the physiologic interplay between the sympathetic and parasympathetic systems, patients may experience symptoms such tachycardia, syncope, palpitations, or dizziness. Proposed acute and chronic mechanisms of autonomic injury in cancer survivors include tumor compression or invasion of autonomic nerves, paraneoplastic effects, side effects of chemoradiation, and deconditioning $[5,9,11,12]$. Autonomic dysfunction is speculated to be an early marker of cardiovascular risk in cancer patients and is associated with an increase allcause mortality $[4,13]$. This highlights the potential survival implications of understanding this condition, in addition to managing the debilitating symptoms patients with autonomic dysfunction often face. Most studies on cardiovascular autonomic dysfunction in cancer survivors focus on the presence or absence of impairment and occasionally grade the severity of autonomic impairment $[7-10,14]$. However, the subtypes and severity of autonomic dysfunction, and associations with specific treatment agents remain poorly understood.

\section{Methods}

\section{Study aim}

The aim of this study is to characterize and quantify the type and severity of cardiovascular autonomic dysfunction in cancer survivors in a cardio-oncology program, and identify associations between malignancies or treatments with cardiovascular autonomic dysfunction.

\section{Study design and setting}

A retrospective analysis of cancer survivors within an academic cardio-oncology program referred for autonomic dysfunction was performed. The electronic medical records were reviewed from January 1st 2010 until December 15th 2019, and study data were extracted. The UCLA institutional review board approval was obtained.

\section{Patient selection}

Patients at least 18 years of age in the cardio-oncology program with a history of malignancy, chemotherapy, or radiation therapy who were referred to the University of California at Los Angeles (UCLA) autonomic testing lab were included in the study. Patients were excluded if they did not undergo autonomic testing or if they had known cardiovascular autonomic dysfunction prior to being diagnosed with cancer.

\section{Data collection}

Patient demographics, symptoms, medical history, cancer-related treatments, echocardiography, cardiodiagnostics, autonomic function test results, and subjective response to autonomic dysfunction directed treatments were collected. Malignancies were classified based on the anatomic location where the majority of the disease was located. In the case of leukemia or diffuse lymphoma, where a particular anatomic location could not be specified, location of disease was categorized as blood and bone marrow. Ventricular function was assessed by echocardiography using left ventricular ejection fraction (LVEF) and myocardial strain. Strain was measured by quantifying global longitudinal strain. Abnormal strain values were based on the age-and-gender adjusted normative values of the software used for interpretation.

\section{Autonomic reflex testing protocol}

Autonomic reflex testing was performed using the Testworks3 System (WR Medical, Minneapolis, MN, USA) or the ANX3.0 platform (ANSAR Medical Technologies, Philadelphia, PA, USA) using standard testing and interpretation protocols [15]. Patients were advised to hold medications that may interfere with the interpretation of results, such as beta-blockers, anticholinergics, and adrenergic antagonists, for $48 \mathrm{~h}$ prior to testing. The patients presented to the autonomic laboratory and were proctored through the four procedures that comprise the autonomic testing protocol: heart rate deep breathing test, Valsalva maneuver, tilt-table test, and sudomotor testing (Additional Table 1 in Additional File 1). Sudomotor testing assesses the integrity of postganglionic peripheral sympathetic nerves that control sweating.

A Composite Autonomic Severity Score (CASS) was assigned to each patient based on their autonomic function [15]. It is the summation of three subdomains: cardiovagal, adrenergic, and sudomotor scores, which are each derived from components of the four autonomic tests. A higher score correlates with a higher degree of autonomic impairment. 


\section{Statistical analysis}

Results are presented as mean, standard deviation (SD), and range.

\section{Results}

Patient demographics, cancer characteristics, and cancerdirected treatments

Of approximately 282 total patients in the cardiooncology program, 24 patients meeting study inclusion criteria were included (Table 1). The 24 patients were referred for suspected cardiovascular autonomic dysfunction based on the presence of typical symptoms or vital sign abnormalities without a definitive alternative cause. Eight patients were female (33.3\%), and the mean age at time of autonomic testing was 51.3 years $(\mathrm{SD} \pm 14.7$, range 25 76). The average duration from cancer diagnosis to autonomic testing was 10.3 years ( $\mathrm{SD} \pm 12.7$, range $0.6-44.1$ ). The reasons for referral for autonomic testing were dizziness $(n=13,54.2 \%)$, tachycardia $(n=13,54.2 \%)$, palpitations $(n=12,50 \%)$, syncope $(n=6,25 \%)$, and dyspnea on exertion $(n=7,29.2 \%)$, with patients having multiple overlapping symptoms in the absence of significant cardiopulmonary disease. The majority of patients had Hodgkin's lymphoma $(n=9,37.5 \%)$ or acute leukemia $(n=5,20.8 \%)$ (Table 2). In terms of anatomic location, most tumors were located in the blood and bone marrow $(n=9,38 \%)$ and mediastinum $(n=9,38 \%)$.

Most patients received radiation therapy to the thorax $(n=10,66.7 \%)$ and neck $(n=8,53.3 \%)$ (Table 3$)$. The most common chemotherapy agents used were alkylating agent $(n=16,66.7 \%)$, vinca alkaloids $(n=13,54.2 \%)$, and anthracyclines $(n=13,54.2 \%)$. Eight patients (33.3\%) received hematopoietic stem cell transplantation. Graft

Table 1 Baseline patient characteristics

\begin{tabular}{ll}
\hline Baseline Patient Characteristics & \\
\hline Demographics & $n=24$ \\
Female (n) & $33.3 \%(8)$ \\
Age at time of autonomic testing, mean years \pm & $51.3 \pm 14.7$ (25- \\
SD (range) & $76)$ \\
Age at time of cancer diagnosis, mean years \pm SD & $40.9 \pm 19.8$ (5- \\
(range) & $70)$ \\
Time since cancer diagnosis to autonomic reflex & $10.3 \pm 12.7(0.6-$ \\
testing, mean years \pm SD (range) & $44.1)$ \\
Time since remission to autonomic testing, mean & $8.0 \pm 12.3(-$ \\
years \pm SD (range) & $0.07-44.0)$ \\
Referral Symptom & $n=24$ \\
Dizziness ( $n$ ) & $54.2 \%(13)$ \\
Dyspnea on Exertion (n) & $29.2 \%(7)$ \\
Palpitations ( $n)$ & $50 \%(12)$ \\
Syncope (n) & $25 \%(6)$ \\
Tachycardia (n) & $54.2 \%(13)$ \\
\hline
\end{tabular}

SD Standard deviation
Table 2 Types and location of diagnosed malignancy

\begin{tabular}{ll}
\hline Cancer characteristics & \\
\hline Primary Malignancy Type & $n=24$ \\
Acute Leukemia (n) & $20.8 \%(5)$ \\
Adenocarcinoma (n) & $8.3 \%(2)$ \\
Aplastic Anemia ( $\mathrm{n})$ & $4.2 \%(1)$ \\
Hodgkin's Lymphoma (n) & $37.5 \%(9)$ \\
Lobular Breast Carcinoma (n) & $12.5 \%(3)$ \\
Multiple Myeloma (n) & $8.3 \%(2)$ \\
Nodal Marginal Zone B-cell Lymphoma (n) & $4.2 \%(1)$ \\
Papillary Thyroid Carcinoma (n) & $8.3 \%(2)$ \\
Rhabdomyosarcoma (n) & $4.2 \%(1)$ \\
Squamous Cell Carcinoma (n) & $4.2 \%(1)$ \\
Location of Majority of Disease & $n=24$ \\
Breast (n) & $17 \%(4)$ \\
Blood and Bone Marrow (n) & $38 \%(9)$ \\
Colon (n) & $4 \%(1)$ \\
Mediastinum (n) & $38 \%(9)$ \\
Neck (n) & $12.5 \%(3)$ \\
Skull (n) & $4 \%(1)$ \\
Stomach (n) & $4 \%(1)$ \\
\hline
\end{tabular}

versus host disease (GVHD) was present in five of these patients at the time of testing (62.5\%). The mean time from hematopoietic stem cell transplant to ARS was 58.1 months ( $\mathrm{SD} \pm 82.3$, range 1.0-237.1).

\section{Autonomic function in cancer survivors}

Twenty-four patients underwent autonomic reflex testing (Fig. 1). Of these patients, 22 (92\%) had evidence of autonomic impairment on autonomic reflex testing. On average, patients demonstrated mild-to-moderate generalized autonomic impairment (CASS $3.6 \mathrm{SD} \pm 2.6$, range $0-9)$ and four patients (16.7\%) had severe generalized autonomic dysfunction. All three domains (cardiovagal, adrenergic, and sudomotor) demonstrated impairment. However, the degree of dysfunction was most pronounced in the adrenergic system (Fig. 2), while more patients had impairment in cardiovagal function albeit minor. The results of individual autonomic tests are reported in Additional Table 2 (see Additional File 1).

Patients were diagnosed with orthostatic hypotension $(n=12,50 \%)$, inappropriate sinus tachycardia (IST) $(n=$ $5,20.8 \%$ ), and postural orthostatic tachycardia syndrome (POTS) $(n=3,12.5 \%)$. Six (25\%) of the patients had a diagnosis of heart failure, with three having previously reduced LVEF and three having preserved LVEF. The majority of patients demonstrated subjective response to pharmacologic autonomic treatments including 
Table 3 Tumor-directed therapeutic interventions

\begin{tabular}{|c|c|}
\hline Tumor-directed therapeutic interventions & \\
\hline Radiation Therapy by Location & $n=15$ \\
\hline Head (n) & $13.3 \%(2)$ \\
\hline $\operatorname{Neck}(n)$ & $53.3 \%(8)$ \\
\hline Thorax (n) & $66.7 \%(10)$ \\
\hline Abdomen (n) & $6.7 \%(1)$ \\
\hline Whole Body (n) & $13.3 \%(2)$ \\
\hline Most Common Chemotherapies by Mechanism of Action & $n=24$ \\
\hline Anthracycline (n) & $54.2 \%(13)$ \\
\hline Anti-Metabolite (n) & $37.5 \%(9)$ \\
\hline Alkylating Agent (n) & $66.7 \%(16)$ \\
\hline Bleomycin (n) & $25 \%(6)$ \\
\hline Microtubule Inhibitor (n) & $66.7 \%(16)$ \\
\hline Platinum-Based DNA Crosslinking (n) & $20.8 \%(5)$ \\
\hline
\end{tabular}

ivabradine, metoprolol, midodrine, and steroids (hydrocortisone and fludrocortisone).

\section{Cardiac evaluation}

On transthoracic echocardiography (TTE), the mean LVEF at time of the autonomic reflex study was $61.2 \%$ ( $\mathrm{SD} \pm 6.9$, range 42.5-75). Three patients had previously reduced LVEF, but all had recovered by the time of autonomic reflex testing. Twenty patients underwent ambulatory cardiac rhythm monitoring. The mean of the average heart rate on ambulatory rhythm monitoring was 90.7 $\mathrm{BPM}$ (SD \pm 13.5 , range 65-117). Fifteen patients completed exercise stress testing. None of the 17 patients had evidence of ischemia with exercise, though two patients (13.3\%) did not achieve adequate workloads and had nondiagnostic tests. Cardiodiagnostic results are reported in Additional Table 3 (see Additional File 1).

\section{Discussion}

The main findings of the present study are: 1) nearly all cancer survivors referred for suspicion of autonomic dysfunction tested positive; 2) dysfunction was demonstrated in all domains of the autonomic nervous system, with particular derangement in the sympathetic system; and 3) patients showed subjective and/or objective symptom response to treatment. These data suggest that cardiovascular autonomic dysfunction may be more prevalent than previously recognized in cancer survivors, and should be screened for, tested, and treated when appropriate.

The results of the autonomic reflex testing suggest that cardiovascular autonomic dysfunction may result predominantly from a systemic process, such as chemotherapy or paraneoplastic syndrome, with a different etiology than sensory peripheral neuropathy caused by many chemotherapy agents. Hematologic disorders, hematopoietic stem cell transplantation, poor functional status, vinca alkaloids, alkylating agents, anthracyclines, chest radiation, and neck radiation were associated with autonomic dysfunction. The majority of patients were diagnosed with orthostatic hypotension, POTS, or IST and had subjective improvement with personalized treatment. To the best of our knowledge, this is the first study to report these findings in cancer survivors with suspected autonomic dysfunction. Clinicians caring for these patients should have a low threshold to refer for autonomic evaluation.

\section{Autonomic function}

Cardiovascular autonomic dysfunction is commonly seen in patients with neurodegenerative disorders and diabetes mellitus [16]. In diabetes mellitus, the mechanism is thought to be due to metabolic and ischemic damage to both the sympathetic and parasympathetic systems [17]. In Parkinson's disease and multiple system atrophy, the underlying mechanism of autonomic impairment is thought to be due to sympathetic

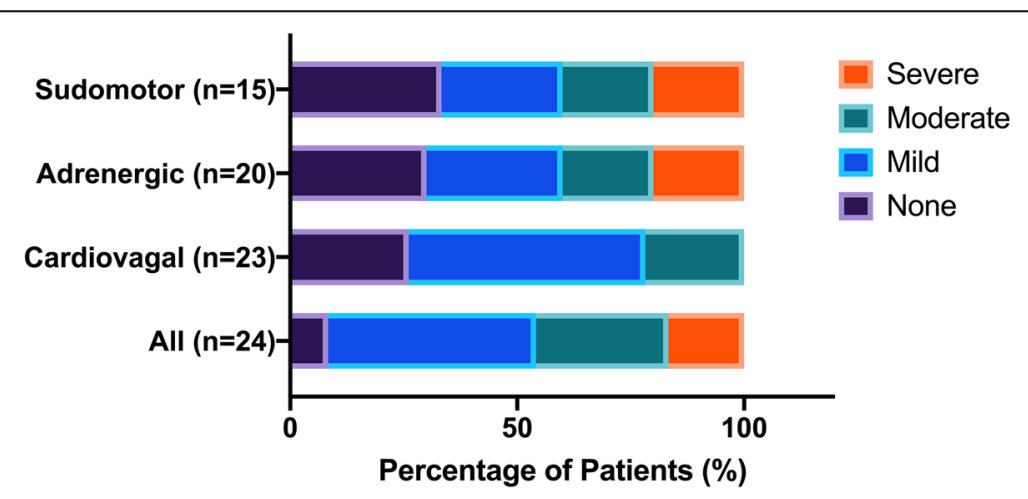

Fig. 1 Composite Autonomic Severity Score (CASS) in Cancer Survivors Diagnosed with Autonomic Dysfunction. The severity and distribution of autonomic dysfunction is quantified using the CASS to assess the sudomotor, adrenergic, and cardiovagal systems. The presence of impairment in any system is reflected by "All" 


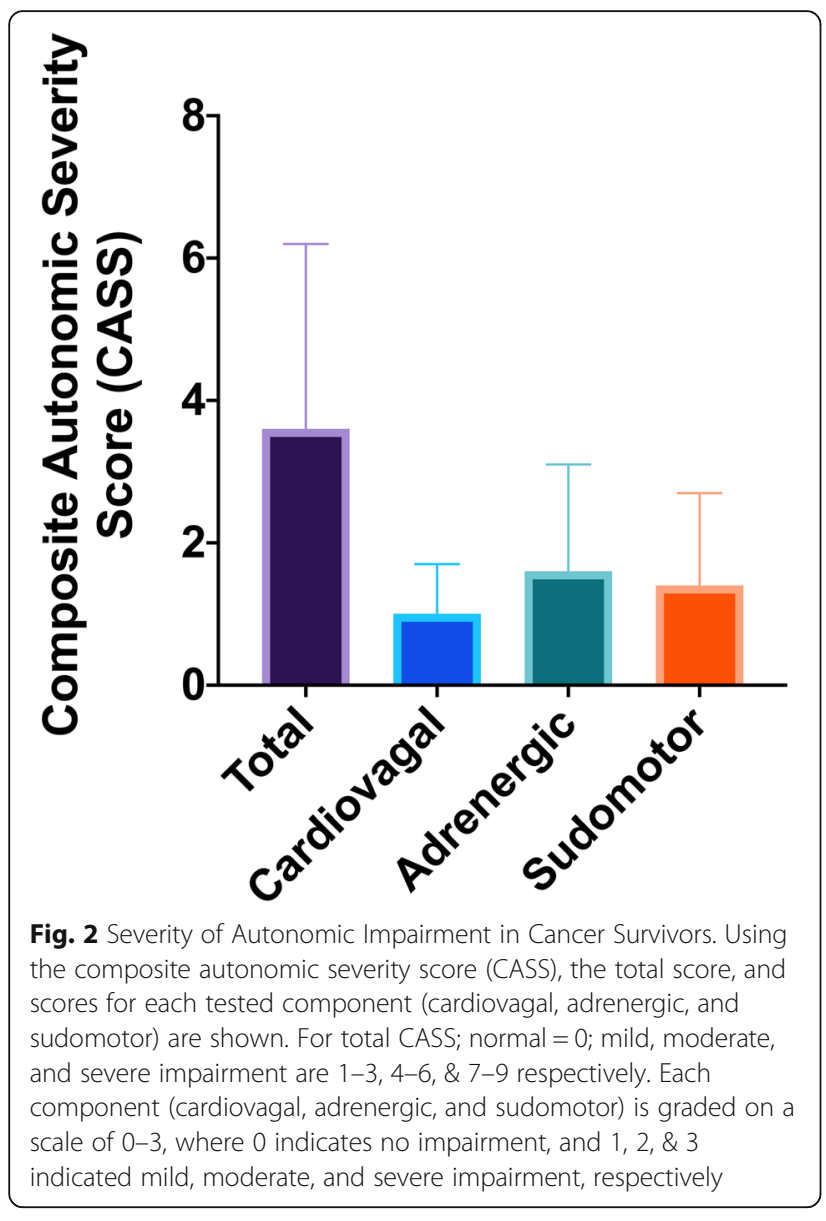

denervation of the heart and vasculature $[18,19]$. The pathologic mechanism of cardiovascular autonomic dysfunction in cancer survivors is poorly understood [5]. In this study, both the parasympathetic and sympathetic arms demonstrated dysfunction, with a greater degree of impairment in the sympathetic nervous system. This phenotype is similar to that seen in multiple system atrophy. Diabetes and Parkinson's disease tend to have more proportionate distribution of impairment between the sympathetic and parasympathetic arms [20-22].

The patients in this study also had derangements on sudomotor testing, which assesses the integrity of postganglionic sympathetic nerves. Moreover, the degree of sudomotor impairment tended to increase with increasing levels of sympathetic and parasympathetic dysfunction. This suggests that autonomic dysfunction in cancer survivors may result from a diffuse process, such as chemotherapy or paraneoplastic effects, as opposed to a local process, such as radiation therapy, tumor invasion, or inflammation surrounding the malignancy.

\section{Patient characteristics}

The heterogeneity in age and time to autonomic testing may reflect a referral bias due to the non-specific nature of these symptoms [5, 12]. It is plausible that patients who were sent for autonomic reflex testing later in their cancer survivorship were symptomatic for an extended period of time. In this case, testing may have been delayed due to low suspicion for autonomic etiology, accessibility issues, or lack of familiarity with autonomic reflex testing. Another explanation is that these findings represent multiple mechanisms of neurotoxicity that vary in time from original insult to clinical presentation. Moreover, patients with obvious, severe autonomic dysfunction or subtle autonomic dysfunction may not have been referred for autonomic reflex testing due to lack of perceived diagnostic utility or low clinical suspicion for autonomic etiology, respectively.

The majority of patients (75\%) had hematologic disorders, which was disproportionately higher than the 9.5\% estimated prevalence among cancer survivors in the United States [23]. Many of the chemotherapy regimens used to treat these disorders, such as CHOP (cyclophosphamide, doxorubicin, vincristine, prednisone) or ABVD (doxorubicin, bleomycin, vinblastine, dacarbazine), include anthracyclines and vinca alkaloids. Given the diffuse pattern of autonomic impairment seen on reflex testing, the association between hematologic malignancies and autonomic dysfunction may be due to paraneoplastic effects of the malignancy or the chemotherapies used in those patients, as opposed to a more localized process such as direct tumor invasion.

Eight of the patients received hematopoietic stem cell transplantation. Deuring et al. found that patients who received hematopoietic stem cell transplantation had higher degree of autonomic dysfunction compared to controls [24]. This may suggest autonomic impairment was either due to graft versus host disease or effects of the myeloablative regimen. Most patients had malignancies either in the neck or thorax or had received radiation treatments in those areas. While the results of the autonomic reflex testing suggest a more diffuse neuropathy, it is possible that local tumor-related effects had some effect given that the autonomic baroreflex pathways are located the neck and thorax as well.

The effects of chemoradiation on peripheral sensory nerves are well established [25]. Given the similarities in myelination between peripheral sensory and autonomic nerves, these mechanisms have been extrapolated to be possible explanations for autonomic dysfunction [25]. The most common chemotherapy agents in this study were vinca alkaloids, alkylating agents, and anthracyclines. There is literature suggesting associations between these agents and autonomic impairment. Microtubule inhibitors, such as vinca alkaloids, are a well-known cause of peripheral sensory neuropathy [25]. The effect on the autonomic system was evaluated by a Roca et al., who found that vinca alkaloids treatment altered orthostatic 
blood pressure and heart rate with deep breathing [26]. While alkylating agents are not commonly associated with neuropathy, a study by Dobrek et al. demonstrated a relationship between alkylating agents and alterations in heart rate modulation [27]. The two patients in our study who received alkylating agents and did not receive microtubule inhibitors, anthracyclines, or platinum-based agents had severe generalized autonomic impairment most prominent in the adrenergic and sudomotor systems. Anthracyclines have well-established association with cardiotoxicity, but not necessarily with neuropathy. Anthracyclines have been associated with abnormalities in heart rate variability [28]. In patients with diabetes, there is some evidence that cardiac autonomic dysfunction is associated with nonischemic cardiomyopathy [29]. The one patient who received anthracyclines, but did not received microtubules or alkylating agents, had moderate autonomic dysfunction with severe adrenergic impairment.

The association between chemotherapy agents, such as anthracyclines and alkylating agents, that are not traditionally associated with neuropathy and cardiovascular autonomic dysfunction suggest that autonomic impairment in cancer survivors may be caused by a different mechanism than the sensory peripheral neuropathy seen with many chemotherapy agents. Moreover, given the number of patients in this study that had cardiomyopathy or received anthracyclines, it is possible that autonomic dysfunction is a precursor to, or at the very least, associated with cardiomyopathy [4].

\section{Clinical implications}

Cardiovascular autonomic dysfunction in cancer survivors is a poorly understood condition that carries significant morbidity and is associated with an increase all-cause mortality [13]. In this study, most patients had either orthostatic hypotension, POTS, or IST, which are recognized by the Heart Rhythm Society as autonomic diseases [30]. Given our limited knowledge on cardiovascular autonomic dysfunction in cancer survivors, there are currently no published guidelines on treatment options [31]. Evidence-based pharmacologic treatment strategies for autonomic dysfunction are largely based on studies of patients with neurodegenerative diseases, diabetes mellitus, and infiltrative diseases [32]. The effectiveness of treatment is generally varied and often poor. In this cohort, the results of autonomic reflex testing were used to tailor personalized, autonomic treatment to each patient. The majority of patients had subjective improvement in treatments, which further demonstrates the utility of this tool.

This study also exemplifies the role of a cardio-oncology program in identifying, preventing, and managing the unique cardiovascular comorbities, such as cardiovascular autonomic dysfunction, faced by this population to improve their overall quality of life. Physicians caring for these patients should have a low threshold to refer for autonomic evaluation, particularly for patients with hematologic malignancies, tumor burden in the neck or chest, cardiomyopathy, or those who have received vinca alkaloids, alkylating agents, anthracyclines, or hematopoietic stem cell transplantation. Autonomic reflex testing may suggest personalized treatment options to improve outcomes and provide an objective measure to assess response to treatment and progression of disease.

\section{Limitations}

This study has several limitations. First, as this study was a retrospective, descriptive case series, statistical hypothesis testing and control groups were not used given the small sample size and large number of variables (cancer types, chemotherapy agents, etc.). There is a selection bias as only symptomatic patients were referred for autonomic reflex testing, which represents a select subset of the total cardio-oncology patient population at this institution. However, given the significantly limited data that exists in this population, particularly among symptomatic patients rather than patient with incidentally found autonomic dysfunction, we believe this information provides useful data for the field. Moreover, there is also a referral bias as the proportion of different malignancies managed and the specific treatment regimens used for different cancers vary per institution. Additionally, as cancer treatments were composed of multi-drug regimens, individual associations were difficult to identify. Furthermore, given the difficulty in determining equipotency between doses and routes of administration between different chemotherapeutic agents, medication use was classified by the presents of administration rather than by cumulative dosing. Autonomic treatments were personalized, which led to a lack of standardization with regard to treatment modality. However, given the paucity of data on this topic, we believe this study provides novel insights into the diagnosis of and management of dysautonomia associated with cancer treatment.

\section{Conclusion}

Cardiovascular autonomic dysfunction is an emerging, but poorly understood topic in cancer survivors. In this cohort study, cancer-directed treatments were associated with cardiovascular autonomic dysfunction. Large prospective studies focusing on confirming these associations and determining the efficacy of individual therapies based on dysautonomic mechanisms and certain cancer treatments are warranted. This will likely improve quality of life metrics and potentially attenuate short and long term comorbidity in this unique and poorly understood patient population. 


\section{Supplementary information}

Supplementary information accompanies this paper at https://doi.org/10. 1186/s40959-020-00065-9.

Additional file 1: Additional Table 1. Autonomic Reflex Testing Interpretation. Additional Table 2. Autonomic Results by Individual Tests. Additional Table 3. Cardiodiagnostic Testing.

\section{Abbreviations}

ABVD: Doxorubicin, bleomycin, vinblastine, dacarbazine; afib: Atrial fibrillation; ARS: Autonomic reflex study; avg: Average; BP: Blood pressure; BPM: Beats per minute; CASS: Composite Autonomic Severity Score;

CHOP: Cyclophosphamide, doxorubicin, vincristine, prednisone; DBP: Diastolic blood pressure; GVHD: Graft versus host disease; HR: Heart rate; HRDB: Heart rate deep breathing; HSCT: Hematopoietic stem cell transplantation; IST: Inappropriate sinus tachycardia; JoVE : Journal of Visualized Experiments; LVEF: Left ventricular ejection fraction; MAP: Mean arterial blood pressure; max: Maximum; METs: Metabolic equivalents; min: Minimum; PASP: Pulmonary artery systolic pressure; POTS: Postural orthostatic tachycardia syndrome; RSA: Respiratory sinus arrhythmia; SBP: Systolic blood pressure; SD: Standard deviation; secs: Seconds; SVT: Supraventricular tachycardia; TTE: Transthoracic echocardiography

\section{Acknowledgements}

None.

\section{Authors' contributions}

BN contributed to study conception and design, acquisition of data, analysis of data, drafting of manuscript, and editing of manuscript: SA contributed to acquisition of data, analysis of data, drafting of manuscript, and editing of manuscript. ML contributed to acquisition of data, analysis of data, and editing of manuscript. EHY contributed to study conception and design, acquisition of data, analysis of data, drafting of manuscript, and editing of manuscript. OAA contributed to study conception and design, acquisition of data, analysis of data, drafting of manuscript, and editing of manuscript. All authors read and approved the final manuscript.

\section{Funding}

None.

\section{Availability of data and materials}

The datasets used and/or analyzed during the current study are available from the corresponding author on reasonable request.

\section{Ethics approval and consent to participate}

The UCLA institutional review board approval was obtained for this study under IRB reference number 19-000771.

\section{Consent for publication}

Not applicable.

\section{Competing interests}

None.

\section{Author details}

'Division of Internal Medicine, Department of Medicine, University of California at Los Angeles, Los Angeles, CA, USA. ${ }^{2}$ UCLA Cardio-Oncology Program and Division of Cardiology, Department of Medicine, University of California at Los Angeles, Los Angeles, CA, USA. ${ }^{3}$ UCLA Cardiac Arrhythmia and Neurocardiology Research Center, David Geffen School of Medicine at UCLA, University of California at Los Angeles, 100 Medical Plaza, Suite 660, Westwood Blvd, Los Angeles, CA 90095-1679, USA.

Received: 27 May 2020 Accepted: 13 July 2020

Published online: 28 July 2020

\section{References}

1. Society AC. Cancer facts \& figures 2019, vol. 2019; 2019.

2. Society AC. Cancer treatment \& survivorship facts \& figures 2019-2021. Atlanta: American Cancer Society; 2019.
3. Hewitt M, Greenfield S, Stovall E. From cancer patient to cancer survivor: lost in transition. Washington, DC: National Academies Press; 2005.

4. Lakoski SG, Jones LW, Krone RJ, Stein PK, Scott JM. Autonomic dysfunction in early breast cancer: incidence, clinical importance, and underlying mechanisms. Am Heart J. 2015;170(2):231-41.

5. Coumbe BGT, Groarke JD. Cardiovascular autonomic dysfunction in patients with cancer. Curr Cardiol Rep. 2018;20(8):69.

6. Stone CA, Kenny RA, Nolan B, Lawlor PG. Autonomic dysfunction in patients with advanced cancer; prevalence, clinical correlates and challenges in assessment. BMC Palliat Care. 2012;11(1):3.

7. Guo Y, Palmer JL, Strasser F, Yusuf SW, Bruera E. Heart rate variability as a measure of autonomic dysfunction in men with advanced cancer. Eur J Cancer Care. 2013:22(5):612-6.

8. Bruera E, Chadwick S, Fox R, Hanson J, MacDonald N. Study of cardiovascular autonomic insufficiency in advanced cancer patients. Cancer Treat Rep. 1986;70(12):1383-7.

9. Walsh D, Nelson KA. Autonomic nervous system dysfunction in advanced cancer. Support Care Cancer. 2002;10(7):523-8.

10. Turner ML, Boland OM, Parker AC, Ewing DJ. Subclinical autonomic dysfunction in patients with Hodgkin's disease and non-Hodgkin's lymphoma. Br J Haematol. 1993;84(4):623-6.

11. Bilora F, Veronese F, Zancan A, Biasiolo M, Pomerri F, Muzzio PC. Autonomic dysfunction in Hodgkin and non-Hodgkin lymphoma. A paraneoplastic syndrome? Hematol Rep. 2010;2(1):e8.

12. Adams MJ, Lipsitz SR, Colan SD, Tarbell NJ, Treves ST, Diller L, et al. Cardiovascular status in long-term survivors of Hodgkin's disease treated with chest radiotherapy. J Clin Oncol. 2004;22(15):3139-48.

13. Groarke JD, Tanguturi VK, Hainer J, Klein J, Moslehi JJ, Ng A, et al. Abnormal exercise response in long-term survivors of hodgkin lymphoma treated with thoracic irradiation: evidence of cardiac autonomic dysfunction and impact on outcomes. J Am Coll Cardiol. 2015;65(6):573-83.

14. Adams SC, Schondorf R, Benoit J, Kilgour RD. Impact of cancer and chemotherapy on autonomic nervous system function and cardiovascular reactivity in young adults with cancer: a case-controlled feasibility study. BMC Cancer. 2015;15:414.

15. Novak P. Quantitative autonomic testing. J Vis Exp. 2011;(53):e2502. https:// doi.org/10.3791/2502.

16. Kaufmann $\mathrm{H}$, Goldstein DS. Autonomic dysfunction in Parkinson disease. In: Handbook of clinical neurology, vol. 117. Amsterdam: Elsevier; 2013. p. 25978.

17. Vinik Al, Maser RE, Mitchell BD, Freeman R. Diabetic autonomic neuropathy. Diabetes Care. 2003:26(5):1553-79.

18. Goldstein DS, Holmes C, Li S-T, Bruce S, Metman LV, Cannon RO III. CardiaC sympathetic denervation in Parkinson disease. Ann Intern Med. 2000;133(5): 338-47.

19. Goldstein DS, Holmes CS, Dendi R, Bruce SR, Li S-T. Orthostatic hypotension from sympathetic denervation in Parkinson's disease. Neurology. 2002;58(8): 1247-55.

20. Low PA, Benrud-Larson LM, Sletten DM, Opfer-Gehrking TL, Weigand SD, O'Brien PC, et al. Autonomic symptoms and diabetic neuropathy: a population-based study. Diabetes Care. 2004;27(12):2942-7.

21. Low PA. Composite autonomic scoring scale for laboratory quantification of generalized autonomic failure. Mayo Clin Proc. 1993;68(8):748-52.

22. Kimpinski K, lodice V, Burton DD, Camilleri M, Mullan BP, Lipp A, et al. The role of autonomic testing in the differentiation of Parkinson's disease from multiple system atrophy. J Neurol Sci. 2012;317(1):92-6.

23. Noone A, Howlader N, Krapcho M, Miller D, Brest A, Yu M, et al. SEER cancer statistics review, 1975-2015. Bethesda: National Cancer Institute; 2018.

24. Deuring G, Kiss A, Halter J, Passweg J, Grossman P. Cardiac autonomic functioning is impaired among allogeneic hematopoietic stem cell transplantation survivors: a controlled study. Bone Marrow Transplant. 2017;52(1):66-72.

25. Quasthoff S, Hartung HP. Chemotherapy-induced peripheral neuropathy. Neurol. 2002;249(1):9-17

26. Roca E, Bruera E, Politi PM, Barugel M, Cedaro L, Carraro S, et al. Vinca alkaloid-induced cardiovascular autonomic neuropathy. Cancer Treat Rep. 1985;69(2):149-51.

27. Dobrek L, Baranowska A, Thor PJ. The influence of oxazaphosphorines alkylating agents on autonomic nervous system activity in rat experimental cystitis model. Acta Pol Pharm. 2013;70(6):1097-105.

28. Tjeerdsma G, Meinardi MT, van Der Graaf WT, van Den Berg MP, Mulder NH, Crijns HJ, et al. Early detection of anthracycline induced cardiotoxicity in 
asymptomatic patients with normal left ventricular systolic function: autonomic versus echocardiographic variables. Heart. 1999;81(4):419-23.

29. Sacre JW, Franjic B, Jellis CL, Jenkins C, Coombes JS, Marwick TH. Association of cardiac autonomic neuropathy with subclinical myocardial dysfunction in type 2 diabetes. JACC Cardiovasc Imaging. 2010;3(12):1207-15.

30. Sheldon RS, Grubb BP, Olshansky B, Shen W-K, Calkins H, Brignole M, et al. 2015 Heart Rhythm Society expert consensus statement on the diagnosis and treatment of postural tachycardia syndrome, inappropriate sinus tachycardia, and vasovagal syncope. Heart Rhythm. 2015;12(6):e41-63.

31. Cuomo JR, Sharma GK, Conger PD, Weintraub NL. Novel concepts in radiation-induced cardiovascular disease. World J Cardiol. 2016;8(9):504-19.

32. Eschlböck S, Wenning G, Fanciulli A. Evidence-based treatment of neurogenic orthostatic hypotension and related symptoms. J Neura Transm. 2017;124(12):1567-605

\section{Publisher's Note}

Springer Nature remains neutral with regard to jurisdictional claims in published maps and institutional affiliations.

Ready to submit your research? Choose BMC and benefit from:

- fast, convenient online submission

- thorough peer review by experienced researchers in your field

- rapid publication on acceptance

- support for research data, including large and complex data types

- gold Open Access which fosters wider collaboration and increased citations

- maximum visibility for your research: over $100 \mathrm{M}$ website views per year

At BMC, research is always in progress.

Learn more biomedcentral.com/submissions 